\title{
HIGH TEMPERATURE ALUMINIUM PLATE COOLING BY WATER IMPINGEMENT: NUMERICAL SIMULATION WITH ADAPTED CFD WATER COOLING MODELS AND EXPERIMENTAL VALIDATION
}

\author{
ROK KOPUN ${ }^{1}$, STEPHAN UCSNIK ${ }^{2}$, DONGSHENG ZHANG ${ }^{3}$, PETER SAMPL ${ }^{3}$, \\ STEPHAN JÄGER ${ }^{2} \&$ REINHARD TATSCHL ${ }^{3}$ \\ ${ }^{1}$ AVL-AST d.o.o., Slovenia \\ ${ }^{2}$ LKR Leichtmetallkompetenzzentrum Ranshofen GmbH, AIT Austrian Institute of Technology, Austria \\ ${ }^{3} \mathrm{AVL}$ List GmbH, Austria
}

\begin{abstract}
This paper outlines the recently improved Computational Fluid Dynamics (CFD) model to simulate the jet impingement quenching cooling process, validated with experimental data. The main application area of the presented method is heat treatment of cast aluminium alloy parts, known as continuous horizontal chill casting process, mostly used in the automotive and aerospace industries, where accurate heat treatment prediction plays an important role in conceptual and thermal analysis. The comparison between measurement data and numerical results has been carried out to simulate the real time jet impingement cooling process of a high temperature aluminium plate using the commercial CFD code AVL FIRETM. Recently, simulation methodology has been applied to cast cooling processes, successfully demonstrating its ability to simulate cooling effects from $500^{\circ} \mathrm{C}$ down to room temperature in a realistic manner. The Eulerian multi-fluid modelling approach is used to handle the boiling flow and the heat transfer between the heated structure and the sub-cooled liquid. While for the fluid region governing equations are solved for each phase separately, only the energy equation is solved in the solid region. Heat transfer coefficients depend on the boiling regimes which are separated by the variable Leidenfrost temperature and the levitation force. Simulation results are compared with available measurement data on different positions along the aluminium plate. The temperature histories predicted by the presented model correlate very well with the provided measurement data at different monitoring locations.
\end{abstract}

Keywords: multiphase, CFD, continuous casting process, Leidenfrost temperature, levitation force.

\section{INTRODUCTION}

Optimization of heat transfer characteristics in automotive, aeronautic and process industries plays an important role in conceptual and thermal analysis. Hence, an efficient heat treatment technique, such as casting, are introduced in order to prevent component failure and to provide long-lasting products. Aluminium alloys are well established materials in those industry branches, therefore accurate heat treatment is essential.

Casting of Aluminium alloys for different semi-finishes for metal sheets is mostly conducted by means of the direct vertical semi-continuous or the continuous horizontal chill casting process. In this process the melt faces direct contact with the billet shaping casting mould. Primary cooling is responsible for the initial solidification of a thin outer shell of the semi-finished ingot. The subsequent energy deduction and ingot cooling happens through a secondary cooling with a time-dependent pulsed/continuous water impingement onto the solidifying outer shell. Material and mechanical properties of such produced alloys strongly depend on the casting process parameters and on the directly linked best suitable cooling conditions. These in turn have a strong influence on the micro-structure of the fast solidifying outer shell as well as of the internal ingot core. 
This paper presents the development, implementation and validation of enhanced computational fluid dynamics (CFD) multiphase models for numerical modelling and simulation of the cooling process of high temperature Aluminum plates by means of direct water impingement. The paper is split into three major sections. Section 2 presents the research and development work related to the CFD modelling of water jet impingement and heat transfer on high temperature Aluminum plates. Section 3 describes the experimental data record of the transient temperature distributions of water cooled high temperature Aluminum plates, and Section 4 shows the comparison of numerical with experimental results for validation of the newly developed CFD cast cooling model

\section{CFD MODELING OF MULTIPHASE PLATE COOLING}

The CFD code AVL FIRETM solves the general conservation equations of mass, momentum and enthalpy plus additional transport equations for turbulence related quantities. The multiphase treatment is based on the Eulerian multi-fluid method that is the most general approach for simulating multiphase flows. The individual fluids (liquid/gaseous phases) are treated as continuous phases with conservation laws applied for each fluid. An ensemble averaging technique is adopted to remove the microscopic interfaces. This leads to macroscopic conservation equations which are analogous to their single-phase counterparts but differ in that a new variable volume fraction and additional exchange terms between the phases are introduced [1].

\subsection{General multiphase CFD framework AVL FIRE ${ }^{\mathrm{TM}}$}

The solution method adopted in AVL FIRETM is based on a fully conservative finite volume approach. In order to offer full flexibility in terms of the structure and topology of the employed computational meshes, AVL FIRE ${ }^{\mathrm{TM}}$ allows for each computational cell to consist of an arbitrary number of cell faces.

All dependent variables for momentum, pressure, density, turbulent kinetic energy, dissipation rate, and scalar quantities are evaluated at the centres of the computational cells. A second-order midpoint rule is used for integral approximation and a second order linear approximation for calculation of any value at the cell-faces. A diffusion term is incorporated into the surface integral source after employment of the special interpolation practice. The rate of change is discretized by using implicit schemes, namely an Euler implicit scheme and a three-time level implicit scheme of second order accuracy.

Various initial and boundary conditions are available to match the setup of any simulation to the real flow problem. To meet the requirements of different application fields, AVL FIRE $^{\mathrm{TM}}$ simulations may be set up in steady or transient (time stepping) mode. Due to the wide range of applicability of the multi-fluid method, the phase-exchange terms are modelled according to the different types of simulation problems, such as e.g. quenching, cavitation, flash boiling, etc.

\subsection{Multiphase water impingement flow and heat transfer modeling}

The Eulerian multi-fluid model considers each phase as interpenetrating continuum coexisting in the flow domain, with inter-phase transfer terms accounting for phase interactions. From the theoretical work of Drew and Passman [1], the ensemble averaged continuity, momentum and energy equations are presented as: 


\subsubsection{Continuity equation}

$$
\frac{\partial(\alpha \rho)_{k}}{\partial t}+\nabla \cdot(\alpha \rho \vec{V})_{k}=\Gamma_{l \rightarrow k},
$$

subject to the compatibility condition

$$
\alpha_{l}+\alpha_{v}=1
$$

where $\alpha, \rho$ and $\vec{V}$ stand for phase volume fraction, density and velocity vector, respectively. The phase mass change rate (in this particular case, boiling) is $\Gamma_{l}$ and subscript $k$ is the phase index $(k=l$ or $k=v)$. Subscripts $v$ and $l$ denote the vapour and liquid phases in the current work.

\subsubsection{Momentum equation}

$$
\frac{\partial(\alpha \rho \vec{V})_{k}}{\partial t}+\nabla \cdot(\alpha \rho \vec{V} \vec{V})_{k}=-\alpha_{k} \nabla p+\nabla \cdot(\alpha \tau)_{k}+(\alpha \rho)_{k} \vec{g}+M_{l k}+\vec{V}_{i} \Gamma_{l \rightarrow k},
$$

with $p, \vec{g}, \tau$ and $\vec{V}_{i}$ representing pressure, gravity vector, stress tensor and interfacial velocity, respectively. The interfacial momentum exchange $\left(\vec{M}_{l k}\right)$ term is given by

$$
\vec{M}_{l k}=\vec{F}_{D}+\vec{F}_{L E V I}
$$

where $F_{D}$ indicates the drag force and $\vec{F}_{L E V I}$ is the levitation force.

\subsubsection{Drag force}

The drag force exerted on the continuous phase is given as

$$
\vec{F}_{D}=\frac{3}{4} \alpha_{d} \rho_{c} \frac{C_{D}}{d_{b}}\left|\vec{V}_{d}-\vec{V}_{c}\right|\left(\vec{V}_{d}-\vec{V}_{c}\right),
$$

where $C_{D}$ is the drag coefficient depending on the droplet Reynolds number, and $d_{p}$ stands for the water droplet diameter.

\subsubsection{Levitation force}

At the quench front of the water jet impingement cooling process considered in the present work, there is a narrow zone of vigorous boiling with massive production of finer droplets. These finer droplets are ejected almost perpendicular from the hot surface at the quench front, causing the liquid to break up into large irregular droplets. To accurately simulate freesurface liquid jet impingement quenching, it is of great importance to account for the repulsion of water droplets and/or film away from the hot surface and consequently, a force $\vec{F}_{L E V I}$, named as levitation force, is exerted on the liquid phase along the quench front. The model proposed by Kim [2] is adopted in this work, with

$$
\vec{F}_{L E V I}=-0.39 C_{L E V I} \frac{2 \mu_{\mathrm{v}}}{\chi \rho_{\mathrm{v}}} \dot{\mathrm{m}} \vec{n}_{w},
$$

where $C_{L E V I}$ stands for the levitation coefficient, $\mu_{v}$ is the vapour phase viscosity, $\dot{m}$ is the mass change rate due to boiling and $\chi$ is the vapour film thickness, which typically in the range of $10-100 \mu \mathrm{m}$. It should be noticed that this kind of levitation force, eqn (6), is also found in the work of Erickson and Williams [3]. The vapour layer causes lifetime extension of Leidenfrost drops, and according to Bianche et al. [4], it is given by 


$$
\chi= \begin{cases}\left(0.75 \frac{k_{v} \mu_{v} \Delta T}{g L \rho_{v} \rho_{l} C a}\right)^{\frac{1}{4}} \sqrt{d_{p}} & d_{p}>C a, \\ \left(\frac{k_{v} \mu_{v} \Delta T}{L \rho_{v} \sigma^{2}} g \rho_{l} d_{p}\right)^{\frac{1}{3}} d_{p} & d_{p} \leq C a .\end{cases}
$$

Here $C a$ is the capillary length, given as

$$
C a=\sqrt{\frac{\sigma}{g \rho_{l}}} .
$$

Fig. 1 shows the graphical implementation of the $\vec{F}_{L E V I}$, where left side of the figure represent the case without the force and where the right side shows the impact of the force (for general and direct casting scenario).
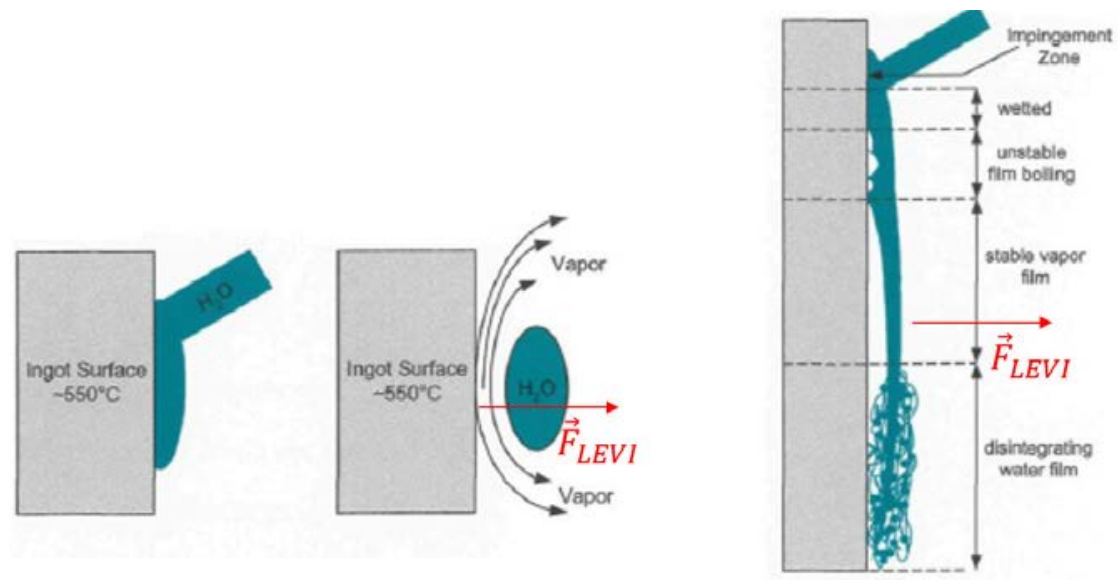

Figure 1: Implementation of $\vec{F}_{L E V I}$ force on general case and direct casting process [5].

\subsubsection{Energy equation}

In previous work [6], [7], vapour bubble condensation in the fluid domain is assumed to be negligible, the phasic static enthalpy equation is then described by

$$
\frac{\partial(\alpha \rho h)_{k}}{\partial t}+\nabla \cdot(\alpha \rho \vec{V} h)_{k}=\nabla \cdot\left(\frac{\alpha \lambda_{T}}{C_{p}} \nabla h\right)_{k}+\frac{3}{4} \alpha_{v} \rho_{l} \frac{C_{H}}{d_{b}}\left(T_{l}-T_{k}\right)+\alpha_{k} \frac{\partial p}{\partial t}+s_{h} .
$$

Thermal conductivity is presented as $\lambda_{T}, C_{H}$ is the inter-phase heat transfer coefficient between phase $k$ and phase $l$ and other terms are lumped into the source term $S_{h}$

$$
S_{h}=\nabla \cdot\left[\frac{\alpha \lambda_{T}}{C_{p}}\left(T-T_{r e f}\right) \nabla C_{p}\right]_{k}+(\alpha \vec{V})_{k} \cdot \nabla \mathrm{p}+(\alpha \tau: \nabla \vec{V})_{k}
$$

\subsubsection{Boiling model}

Based on the assumption that the boiling heat transfer rate is proportional to the phase change rate, the mass transfer predominantly controls the heat transfer. Thus, the phase change rate due to boiling can be written as

$$
\Gamma_{c}=\frac{C_{m} C_{b o i l} h_{b o i l}\left(T_{w}-T_{s a t}\right)}{H_{f g}} \frac{6 \alpha_{v}}{d_{b}}
$$


where $C_{m}, C_{b o i l}, h_{b o i l}$ and $H_{f g}$ are the closure coefficient, the boiling correction coefficient, the boiling heat transfer coefficient and the latent heat of vaporization, respectively. The following sub-sections describe the models applied for heat transfer coefficient $h_{\text {boil }}$ at the different boiling regimes.

\subsubsection{Film boiling model}

Bromley's model [8], originally applied for a horizontal tube, is employed to predict the film boiling heat transfer coefficient $h_{\text {boil }}$

$$
h_{\text {boil }}=0.62\left\{\frac{\lambda_{v}^{3} \rho_{v}\left(\rho_{l}-\rho_{v}\right) g\left[H_{f g}+0.4 C_{p, v}\left(T_{w}-T_{s a t}\right)\right]}{\mu_{v}\left(T_{w}-T_{s a t}\right) d_{b}}\right\}^{0.25},
$$

where $d_{b}$ is the length scale (here the vapour bubble diameter), $\lambda_{v}$ is the vapour thermal conductivity and $C_{p, v}$ stands for the vapour specific heat.

2.2.8 Transition boiling mode

The heat transfer coefficient, $h_{\text {boil }}$ in the transition boiling regime is given by

$$
h_{\text {boil }}=\frac{q_{T B}}{T_{w}-T_{\text {sat }}}
$$

where the heat flux in transition boiling regime qTB is computed as follows

$$
q_{T B}=\phi q_{C H F}+(1-\phi) q_{M H F} .
$$

In eqn (14), $q_{C H F}$ denotes the Critical Heat Flux, and $q_{M H F}$ the Minimum Heat Flux. In a relation to the Zuber's correlation [9], the critical heat flux is computed as

$$
q_{C H F}=0.131 \rho_{v} H_{f g}\left[\frac{\sigma g\left(\rho_{l}-\rho_{v}\right) g}{\rho_{v}^{2}}\right]^{0.25},
$$

where $\sigma$ is the surface tension of the liquid phase. The Minimum Heat Flux correlation for the quenching system in eqn (14) is

$$
q_{M H F}=0.09 K_{M H F} \rho_{v} H_{f g}\left[\frac{g\left(\rho_{l}-\rho_{v}\right)}{\rho_{l}+\rho_{v}}\right]^{0.5}\left[\frac{\sigma}{g\left(\rho_{l}-\rho_{v}\right)}\right]^{0.25} .
$$

$K_{M H F}$ serves as a model parameter and its typical value varies from 1 to 15 . Further, more detailed information about the applied quenching model can be obtained from AVL FIRETM multi-fluid model solver theory guide [10] and the reference [11].

\subsubsection{Quench front temperature}

During free-surface liquid jet impingement quenching, the quench front propagates in the direction of flow along the surface. Heat transfer occurs by boiling (nucleate, transition or film boiling) and/or single-phase forced convection upstream of the quenching front, while conduction and radiation heat transfer take place at dry zones of the surface downstream of the front. A transition between the boiling and conduction-radiation zones takes place when the surface temperature first drops below the quench front temperature. Quench front temperature is defined as the highest solid surface temperature when film boiling is taking place on the heated surface. Here, the correlation presented by Brenson [12] is employed to evaluate the quench front temperature. It is given by

$$
T_{Q F T}=T_{M F B, B}+\left(T_{M F B, B}-T_{l}\right) \sqrt{\frac{\rho_{l} C_{p, l} \lambda_{l}}{\rho_{s} C_{p, s} \lambda_{s}}}
$$


Based on Taylor instability theory, the minimum film boiling temperature $T_{M F B, B}$ reads

$$
T_{M F B, B}=T_{\text {sat }}+0.127 \frac{\rho_{v} h_{f g}}{\lambda_{v}}\left(\frac{g\left(\rho_{l}-\rho_{v}\right)}{\rho_{l}+\rho_{v}}\right)^{2 / 3}\left(\frac{\sigma}{\left(\rho_{l}-\rho_{v}\right)}\right)^{1 / 2}\left(\frac{\mu_{v}}{g\left(\rho_{l}-\rho_{v}\right)}\right)^{1 / 3} .
$$

\section{PLATE COOLING SET-UP AND RESULTS}

The generation of temperature data during water jet impingement cooling was conducted using a special sensor-equipped test setup [13]. This test setup allows for the horizontal heating of metal coupon plates, the quick transfer from the oven to the impingement cooling to subsequently run the water cooling tests at defined water cooling parameters. Fig. 2(a)-(c) show the setup of the experimental test device.

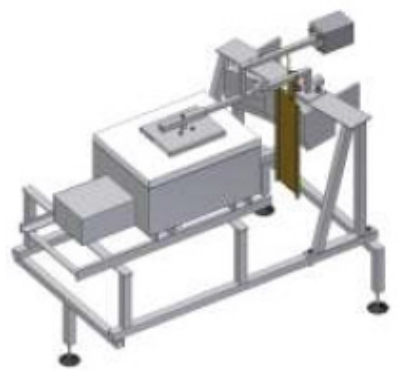

(a)

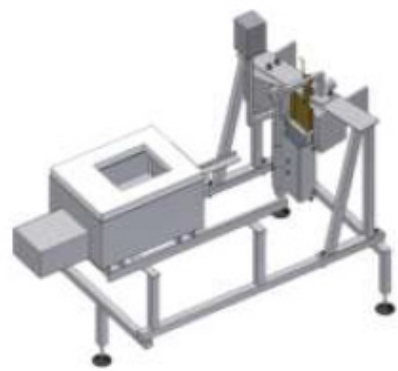

(b)

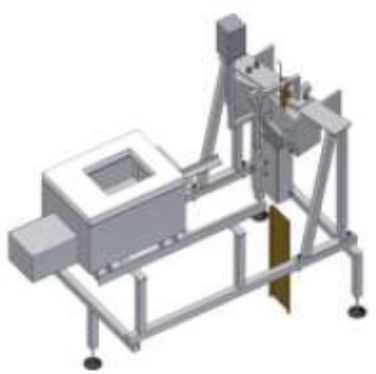

(c)

Figure 2: Experimental setup of the cooling device used for plate cooling tests. (a) Phase of coupon plate heating; (b) Phase of transfer to cooling position; and (c) Removal of shielding plate (brown) [13].

The setup is capable of varying the plate temperature in the oven as well as changing water cooling parameters such as mass flow, flow shape (pulsed, continuous) etc. at the cooling side. For the present investigations the cooling setup was further modified so to also vary the impingement angle and record its influence on the temperature graphs.

For the reference investigation, AA7075 Aluminium billets were vertical chill casted. Out of these billets, five rectangular coupon plates were manufactured (Fig. 3(a)). Dimensions of the coupon plates were $20 \times 100 \times 200 \mathrm{~mm}$. The plates were equipped with 10 thermo-couples along the centre line, $3.0 \mathrm{~mm}$ under the impinged surface of the water-cooled plate (Fig. 3(b)). Thermo-couples T1-T2 were above the impingement zone IZ. T3 was at the IZ and remaining T4-T10 were positioned below the IZ. The coupon plates were heated up to $475^{\circ} \mathrm{C}$ and then transferred to the water cooling. For water cooling, room temperature water was used, the mass flow was continuous between $6-141 / \mathrm{min}$ and the splashing angle was between $11-23^{\circ}$ down from the vertical. During the cooling the specimen faced no movement or transfer. The coupon plate remains vertically arranged in position. Only the cooling water rinses down the cooled surface.

The main function of the whole equipment is to guarantee the reproducible heating and cooling condition for the tests. This will secure the data (temperature) record which will be used for later comparison with numerical simulation results. 


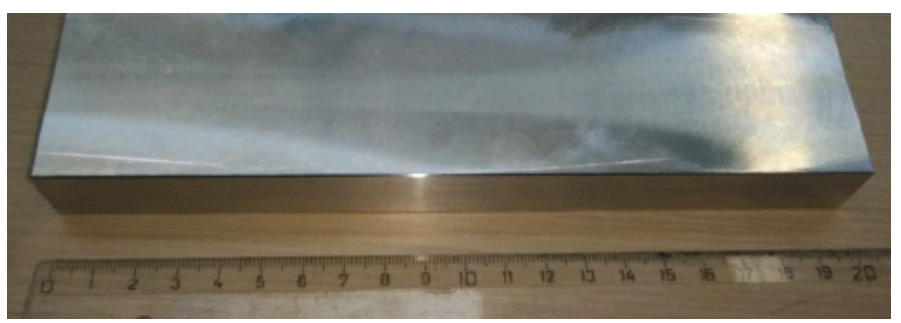

(a)

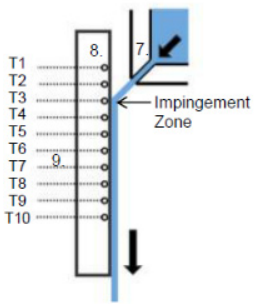

(b)

Figure 3: (a) AA7075 coupon plate as cast with milled surfaces; and (b) Water cooling schema [12].

\subsection{Experimental results}

Fig. 4 shows the temperature results of test scenario A01 for thermo-couples T1-T10 over time. The graphs show an initial maximum temperature plateau at $475^{\circ} \mathrm{C}$ which is followed by the temperature decrease due to water impingement cooling. Thermo-couples $\mathrm{T} 1$ and $\mathrm{T} 2$ show a very smooth graph with the lowest temperature gradients for cooling as these points of the plate do not face direct contact with the cooling water. T3 (green) is directly at the impingement zone and faces the highest temperature gradient during water impingement in the initial face and goes down to $250^{\circ} \mathrm{C}$ within 5 seconds.

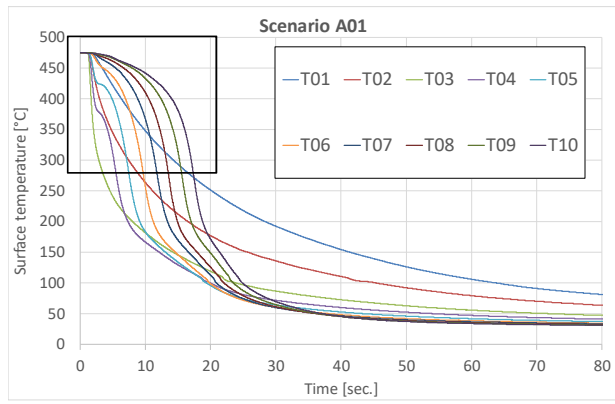

(a)

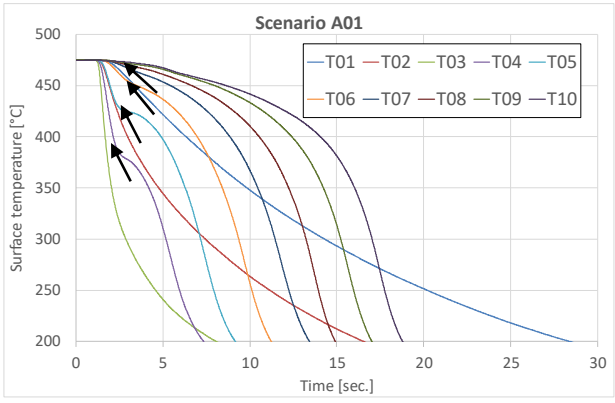

(b)

Figure 4: (a) Temperature results of thermo-couples T1-T10 over time for cooling test scenario A01; and (b) Detail of T-t graphs for time interval 0-30 seconds with indicators for rewarming due to Leidenfrost isolation effect.

The initial cooling gradients of the remaining thermo-couples T5-T10 are smaller compared to T4. In addition, it is visible that these thermo-couples record a temporary delay of the cooling. These are indicated in Fig. 4(b). The delay of the cooling is due to the Leidenfrost effect. This builds a temporary isolation layer between cooling water and the hot coupon plate which rehashes the surface due to internal billet core energy conduction.

\section{NUMERICAL MODELLING OF IMPINGEMENT WATER COOLING PROCESS}

Fig. 5(a) displays the physical domain and the boundary conditions applied in the AVL FIRETM CFD simulation. In the current study, one initial temperature and coolant mass flow 
rate for jet impingement quenching is investigated. For model simplification and faster simulation time, wall symmetry conditions are adopted. The total number of cells for the cooling plate piece (Fig. 5(b)) is about 350,000, with the solid domain consisting of approximately 25,000 cells.

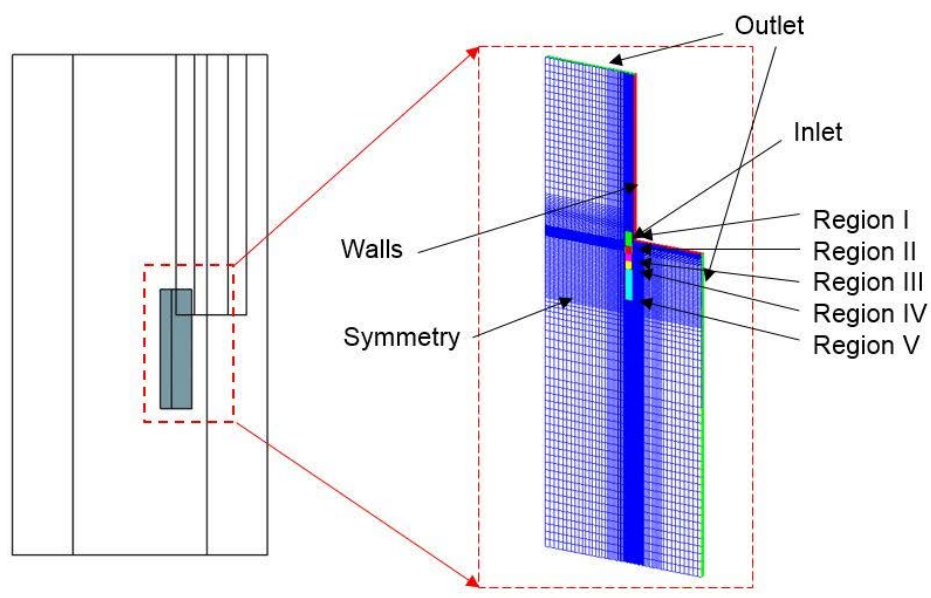

(a)

(b)

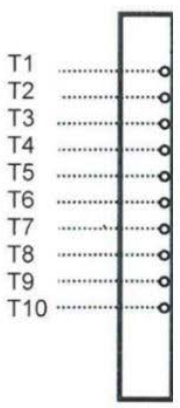

(c)

Figure 5: Physical domain. (a) Boundary conditions; (b) Temperature monitoring points; and (c) For investigated water impingement plate cooling configuration.

With reference to the liquid domain, prescribed atmospheric pressure is exerted at the outlet boundary on the top (green surface), and the inlet section is the given velocity boundary condition from the drilled hole at the side (black surface). In the current simulations, a constant impingement velocity of $14.14 \mathrm{~m} / \mathrm{s}$ is used until the temperature of the quench piece reaches $23^{\circ} \mathrm{C}$. The domain walls at the back and front are treated as adiabatic, whereas symmetry is applied on the sides. Temperature measurements along the presented piece were performed at different positions, and they are referred as T1, T2, T3, T4, T5, T6, T7, T8, T9, and T10, as shown in Fig. 5(c)).

The impingement area is divided into 5 regions according to the different cooling regimes, as proposed by Kim [2] (Fig. 5(b)). Region I is the region above the impingement zone. Region II and Region III are the regions where liquid hits the heated surface, whereas the Region IV and Region V are covering the parts bellow. Each region has its own quenching cooling parameters such as Leidenfrost Limit, Leidenfrost Variable Range, Transient Minimum Heat Flux Factor, Dry Phase Packing Limit and Transient Critical Heat Flux Factor. Details regarding water cooling parameters can be extracted from the AVL FIRETM solver theory guide [10].

\subsection{Numerical results}

The liquid volume fraction and temperature distribution within the structure at different time instants are displayed in the Fig. 6. It can be seen that due to upcoming inlet velocity and newly introduced levitation force, coolant is pushed away from the heated plate, see Fig. 6(a), (b). 


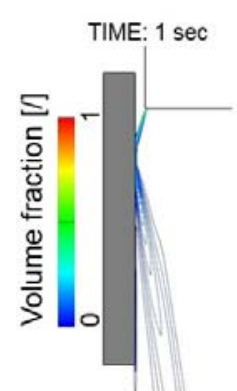

(a)

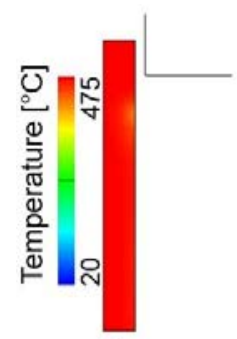

(f)

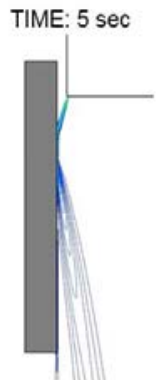

(b)

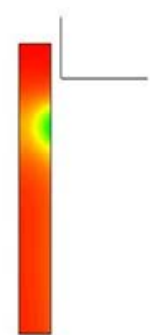

(g)

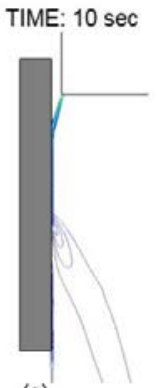

(c)

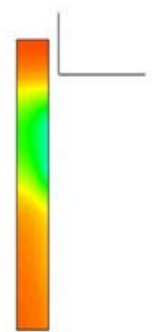

(h)

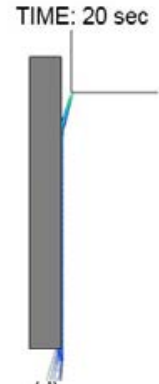

(d)
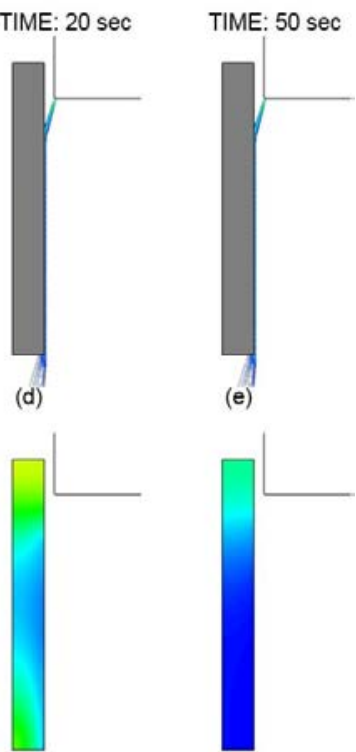

(i)

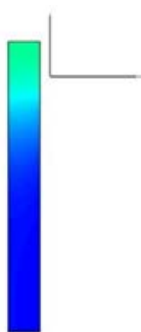

(j)

Figure 6: Water jet cooling of $475^{\circ} \mathrm{C}$ plate at different time steps; water volume fraction, resulting temperature distribution in the plate.

The solid plate starts to cool down short after the coolant hits the plate. At the time of $5 \mathrm{~s}$, the surface temperature at the impingement zone is already around $360^{\circ} \mathrm{C}$, whereas the other part of the solid plate still shares the temperature close to initial value, Fig. 6(g). As soon as the surface temperature drops below the Leidenfrost temperature, transition boiling and later nucleate boiling take place. This effect is visible in Fig. 6(d), (e), where due to lower solid temperature, levitation force is no longer active and that the coolant is no longer pushed away from the heated surface. This results in a faster cooling process. At the time of $20 \mathrm{~s}$, it is observed in Fig. 6(i), that the impingement zone is already cooled down to ambient temperature, whereas the upper and lower parts of the solid still share higher temperature. A uniform cooling temperature is reached at the time of $50 \mathrm{~s}$ (see Fig. 6(j)). The newly implemented Levitation force is well visible on the Fig. 6(a)-(c), where some amount of water stays on the surface and cool down the solid plate, whereas the rest of the water is rebounced from the heated surface, due to newly implementation. When the temperature falls below the Leidenfrost temperature, levitation force no longer visible, and all water which is entering the system stayed on the cooling plate (no re-bouncing effect is visible anymore see Fig. 6(d), (e)).

\subsection{Cooling model validation}

The model capability to predict quench rates is validated through comparison of measured and numerically predicted temperature histories (see Fig. 7). From all monitoring points the simulated film and transition boiling regime are well described with the adopted jet impingement quenching model, where very good agreement with the available measurement data is achieved. 


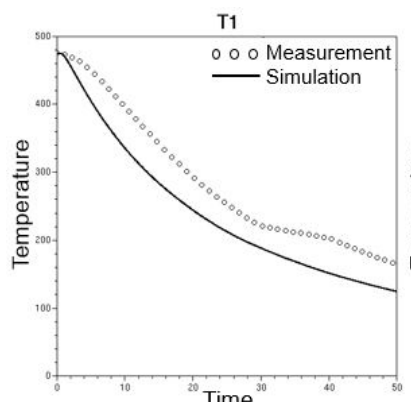

(a)

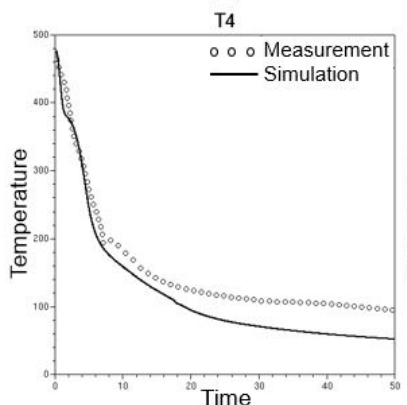

(d)

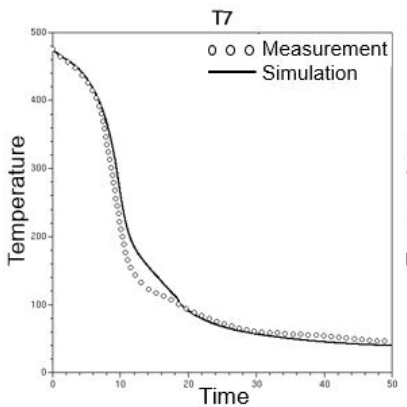

(g)

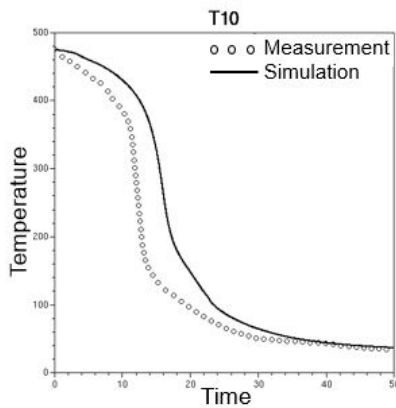

(j)

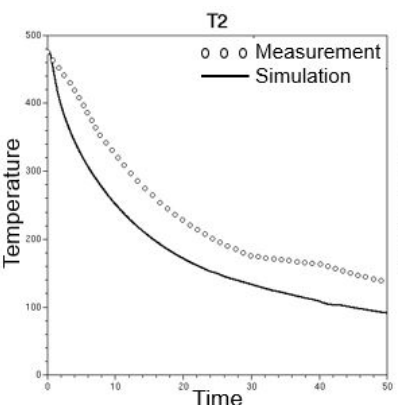

(b)

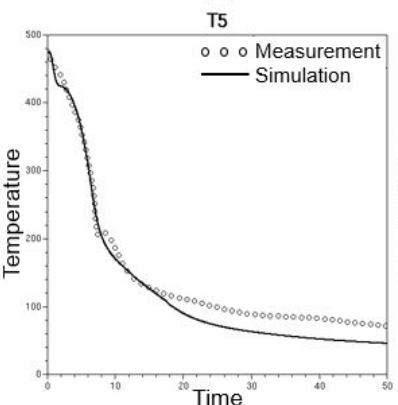

(e)

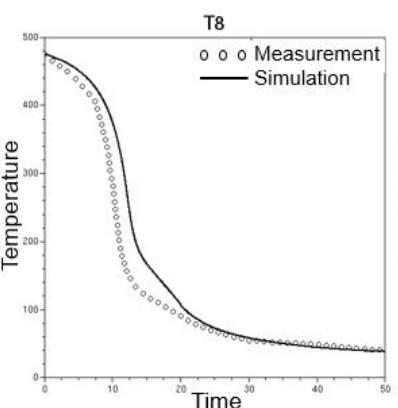

(h)

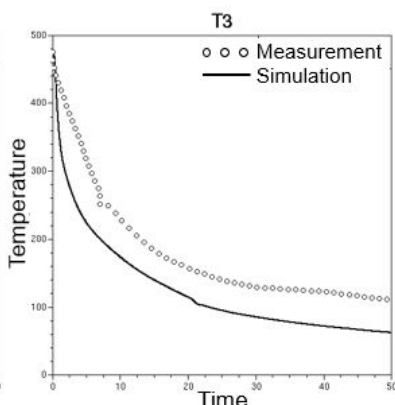

(c)

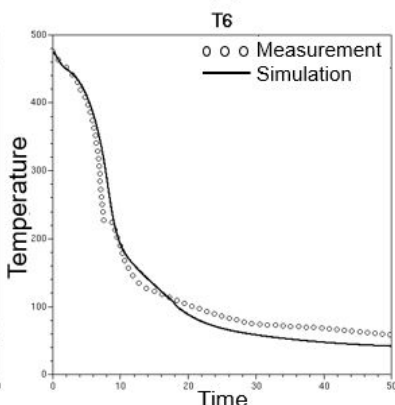

(f)

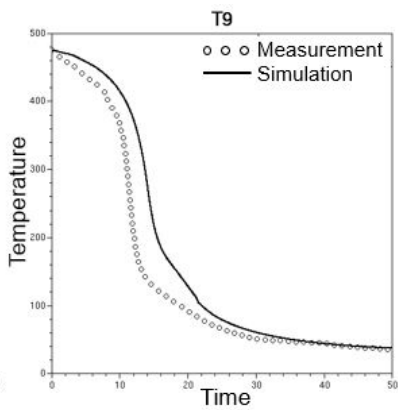

(i)

Figure 7: Comparison of numerically predicted and measured solid part temperature for jet impingement quenching model with water temperature of $20^{\circ} \mathrm{C}$ at monitoring points T1, T2, T3, T4, T5, T6, T7, T8, T9 and T10 


\section{SUMMARY}

For improvement of the prediction of transient temperature distribution evolution during the vertical chill casting process of Aluminium alloys in the initial starting phase, it is essential to adopt highly accurate models for describing the heat flux away from cast billets, especially in the top hot solidifying outer billet shell at the phase of water impingement.

This paper presented the development, implementation and validation of an enhanced CFD multiphase heat transfer model in AVL FIRETM for numerical modelling and simulation of the cooling process of high temperature Aluminium plates by means of direct water impingement. For this purpose, the AVL FIRETM multiphase model was adapted and extended in order to capture the specific phenomena characteristic for high temperature Aluminum plates hit by cold water (e.g. liquid phase levitation, appearance of different boiling regimes, Leidenfrost effect, etc.). The resulting model was applied to a real test case scenario of water cooled coupon plates made of AA7075. The comparison of the numerical simulation results with recorded temperature data at selected thermo-couple positions shows good agreement between the numerical model and the test data especially for the points at and below the impingement zone. With the current implementation user has to define the impingement region out of existing model, but in future we would like to establish automatic approach, where the solver would recognize those regions, and automatically divide this into five regions and set all the needed data for simulation.

The newly developed model in AVL FIRETM sets the basis for future research work so to exchange the generated transient heat flux with numerical finite element models of the continuous vertical chill casting process and to improve the temperature distribution especially in the starting phase of the process.

\section{ACKNOWLEDGEMENTS}

The authors would like to thank the Austrian Research Promotion Agency (FFG), the Federal Ministry for Transport, Innovation and Technology (BMVIT) and the State of Upper Austria for sponsoring this research work in the framework of COMET project Amoree. The authors would also like to acknowledge the support of the AMAG AG and of the Heat Transfer and Fluid Flow Laboratory, Brno University of Technology for conducting the experimental plate cooling tests.

\section{REFERENCES}

[1] Drew, D.A. \& Passman, S.L., Theory of Multi-Component Fluids, Springer: New York, 1998.

[2] Kim, H., Floating phenomenon of a water drop on the surface of liquid nitrogen. $J$. Korean Physical Society, 49, pp. 1335-1338, 2006.

[3] Erickson, D.W. \& Williams, G.A., Experimental studies of the cryogenic Leidenfrost effect. http://reu.physics.ucla.edu/common/papers/2005/david_erickson.pdf. Accessed on: 14 Apr. 2019.

[4] Bianche, A.-L., Clanet, C. \& Quéré, D., Leidenfrost drops. Phys. Fluids, 15(6), pp. 1632-1637, 2003.

[5] Meenken, T., MSc thesis, Université du Québec à Chicoutimi, Canada, 2002.

[6] Kopun, R., Škerget, L., Hriberšek, M., Zhang, D.S., Stauder, B. \& Greif, D., Numerical simulation of immersion quenching processes for cast aluminum part at different pool temperatures. Appl. Therm. Eng., 65, pp. 74-84, 2014.

[7] Srinivasan, V., Moon, K., Greif, D., Wang, D.M, \& Kim, M., Numerical simulation of immersion quench cooling process using an Eulerian multi-fluid approach. Appl. Therm. Eng., 30, pp. 499-509, 2010. 
74 Computational and Experimental Methods in Multiphase and Complex Flow X

[8] Bromley, L.A., Heat transfer in stable film boiling. Chem. Eng. Progr., 46, pp. 221227, 1950.

[9] Zuber, N., On the stability of boiling heat transfer. Trans. ASME, 80, 1958.

[10] AVL LIST GmbH, FIRE CFD Solver, Eulerian multi-fluid model. Solver Theory Guide, Graz, Austria, 2018.

[11] Kopun, R. \& Škerget, L., Experimental and numerical investigation of boiling flow in a vertical pipe with phase change using multi-fluid modelling approach. 9th International Conference on Advances in Fluid Mechanics, 2012.

[12] Brenson, P., Film boiling heat transfer from a horizontal surface. ASME J. of Heat Transfer, 83, pp. 351-358, 1961.

[13] Guzej, M., Bartuli, E. \& Kristof, O., Water jet cooling of Aluminum alloy. Proceedings of Metal, Brno, Czech Republic, 2015. 